\title{
The Case for the Cost-effectiveness Analysis of Contextual HIVIAIDS Interventions and the Extent to which it is Relevant to South Africa
}

\begin{abstract}
Josue Mbonigaba
University of KwaZulu Natal

E-mail:mbonigaba@ukzn.ac.za

Doi:10.5901/mjss.2013.v4n3p743

Abstract

Uncertainty and importance of CE evidence of health care interventions more generally, and of HIVIAIDS interventions in particular have been two main reasons put forward to justify the need for cost-effectiveness analysis (CEA) of such interventions. However, CEA focused more on HIVIAIDS interventions, characterized by different set of activities and less on contextual HIVIAIDS interventions (CHIS), characterized by different contexts of implementation. Has the irrelevance of uncertainty and importance argument to CHIs been the reason for limited CEA of such interventions? Using uncertainty and importance of CE evidence arguments which have been used to motivate the conduct of CEA, this paper argues the case for CEA of CHIs and assesses the extent to which this case is relevant to South Africa. The paper finds that the case is strong and that it is relevant to South Africa to a great extent. It recommends more CEA of CHIs for the sake of efficiency-related policy advancement particularly in South Africa.
\end{abstract}

Keywords: Contextual, Cost, effectiveness, CEA of CHI, South Africa, HIVIAIDS, interventions, CHI

\section{Introduction}

The CEA in health care is defined as a systematic inventory of costs and health outcomes of alternative ways of responding to a health problem (Drummond et al., 2005). CEA of health care interventions has been underpinned by a number of arguments that relate mainly to uncertainty around costs, health outcomes of various interventions, and the importance of cost-effectiveness (CE) evidence for efficiency-related policy making (Muennig, 2008). In the HIVIAIDS sector these arguments were also at centre stage of CEA of HIVIAIDS interventions although CEA focused on alternatives HIVIAIDS interventions although each with a different set of activities and not on CHIs. This paper argues the case for the CEA of CHIs.

In this paper, a CHI refers to an HIVIAIDS intervention and the context of its implementation. Specifically, CHIs refer to the same HIVIAIDS intervention in different contexts. The same HIVIAIDS intervention in different contexts results in different CHIs because of the distinct interaction between that HIVIAIDS intervention and each context. For instance, pair prevention of mother-to-child transmission (PMTC) and its urban context are assumed to be distinct PMTCT interventions because of the distinct interaction between PMTCT and each context.

While CEA of HIVIAIDS interventions has been justified by its potential to reduce uncertainty and by the importance of CE evidence from alternative HIVIAIDS interventions, the extent to which arguments relating to uncertainty and the importance of CE evidence support the case for CEA of CHIs has not been assessed. This paper argues the case for CEA of CHIs based on the reasons which justify CEA and assesses the extent to which CEA of CHIs is relevant to South Africa. The paper is organized as follows: In section 2, the case for CEA of CHIs is argued. Section 3 shows the extent to which the case is relevant to South Africa while section 4 discusses the findings and concludes the study.

\section{The case for CEA of CHls}

Arguing the case for CEA of CHIs requires showing that arguments which substantiate the CEA of HIVIAIDS interventions apply to CHIs. Uncertainty around the costs and health outcomes of alternative HIVIAIDS interventions, and the importance of related CE evidence to policy makers, are two main arguments which motivated CEA of such interventions. In making the case for the CEA of CHIs, arguments which supported CEA of HIVIAIDS interventions need to be valid also for the CEA of CHIs. In other words, to defend the case for CEA of CHIs, it must be shown that without such analysis, policy makers will remain uncertain about how cost-effectiveness of $\mathrm{CH}$ ls compare and will miss out on the important CE evidence needed for efficiency-related policy-making. The case is defended starting with the uncertainty argument. 


\subsection{The uncertainty argument}

The uncertainty argument has been used to justify CEA of HIVIAIDS interventions because the activity of an HIVIAIDS intervention can result in more than one health outcome. In most case, each outcome resulting from an HIVIAIDS intervention procedure, determines the next set of activities of that intervention, making different HIVIAIDS interventions most likely to result in different health outcomes and costs. The uncertainty surrounding the costs and health outcomes are confusing for policy makers having to choose between competing interventions, therefore making CEA necessary. CEA was particularly useful in estimating the average costs and health outcomes (expected values) of each alternative, providing policy makers with certain costs and health outcomes on which to base their decisions. For this argument to hold for CEA of CHIs, it must be shown that without CEA of CHIs, policy makers will face uncertainty about the costs and HIV outcomes of $\mathrm{CHIS}$. This can be shown in three types of uncertainties: uncertainty regarding the impact of a given context on HIV outcomes, uncertainty about effectiveness HIVIAIDS interventions in a given context, and uncertainty about costs of HIVIAIDS intervention in a given context.

\subsubsection{Uncertainty about the context's effects on HIV/AIDS outcomes}

The uncertainty about the cost-effectiveness of $\mathrm{CHIs}$ faced by policy makers stems from uncertainty with which a context influences the impact of HIVIAIDS or in other words the uncertainty with which the context influences HIVIAIDS outcomes. The uncertainty with which a context influences the impact of HIVIAIDS (new infections, sickness and deaths) originates from the uncertainty about which factors influence the impact most in that context because many theoretical factors are involved. A theory which has recently dominated the literature in explaining the impact of illness is the theory of socio-economic determinants of health (Marmot \& Wilkinson, 2005) which ties the impact of illness to socio-economic factors. In this theory, socio-economic position in which the individual lives is characterized by levels of income, education and gender relationships which act as determinants of health through material circumstances, psychosocial and behavioural factors, access to health and social cohesion (Valentine \& Solar, 2011; Marmot \& Wilkinson, 2005). The predictions of the theory are shown in Figure 1.

Figure 1. Model of the socio-economic determinants of health outcomes

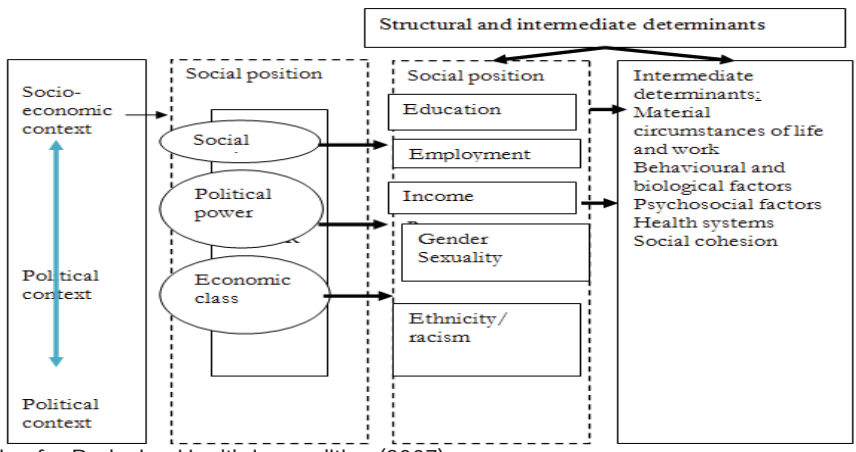

Source: Spanish Commission for Reducing Health Inequalities (2007)

With respect to HIVIAIDS, the theory of socio-economic determinants of health status predicts that socio-economic contexts of lower status are associated with lower income levels to respond to HIVIAIDS, lower levels of knowledge about HIVIAIDS and as an implication more risky behaviour such as injecting drugs and practicing unprotected sex. A lower socio-economic position is also associated with environmental hazards, which cause stressors which in turn lead to drug addiction, alcoholism and consequently the risk of HIVIAIDS infection. In other words, the theory predict that the impact of HIVIAIDS in terms of new infections, AIDS cases and deaths at an individual level is on average higher in contexts with lower socio-economic status.

Theoretical formulations related to the theory of socio-economic determinants of health have tended to focus on specific factors such as cultural factors, stating that an unfavorable HIVIAIDS cultural environment is expected in contexts with lower socio-economic status (Airhihenbuwa, 2004). Other models such as social capital theory have focused on social cohesion. Social capital theory explains that social cohesion, trust and networking help in achieving common health goals (Diclemente et al., 2002). In the case of HIVIAIDS, social capital facilitates the reduction of stigma and as a result, 
the impact of HIVIAIDS is reduced in contexts in which greater social capital exists

At first glance, the theory of the socio-economic determinants of health appears to contradict the uncertainty of the impact of contexts on HIVIAIDS outcomes since one can predict what would be the pattern of HIVIAIDS outcomes from a socio-economic context of lower status to a socio-economic context of higher status. Some empirical evidence also exists to support the prediction of the theory. For example, Snelling et al., (2007) showed that countries in which women had greater knowledge about HIVIAIDS had also less risk behaviours and less severity of HIVIAIDS among women than countries in which HIVIAIDS knowledge was limited. Lower health outcomes among the poorest segment of the populations have also been documented by Bowden et al. (2006) in the United States. Yet, the uncertainty in the theory prediction manifests in the counter examples by Zanakis et al.(2007:1811) who showed that lower HIV indicators were found in countries with higher Gross National product, by Cogneau and Grimm (2006) who found in Cote D'Ivoire greater level of risky behaviours among the well-off segments of the population and Tiruneh (2009) who found higher risk behaviours in better-off countries of Southern Africa than in worse -off countries in the south of Sahara.

Furthermore, even if the pattern of the impact could be predicted higher in a context of lower socio-economic status than in a context of higher socio-economic status, the uncertainty regarding the extent in the difference of the impact would remain. This is because factors underlying the differences in the impact of HIVIAIDS in one pair of contexts, say a context with a low socio-economic status and a context with a high socio-economic status, might be different from the factors underlying the difference of the impact of HIVIAIDS in another pair of contexts with a low socio-economic status and a high socio-economic status. For instance, while higher alcohol abuse might be responsible for the difference in the impact of HIVIAIDS in a pair of a low status socio-economic context and a high status socio-economic context, in another pair of a low status socio-economic context and a high status socio-economic context, gender violence might be the main factor. In this respect, Ferry et al. (2006) provided evidence by showing that different parameters may be underlying the observed impact. Even where the same factor, say gender violence, underlies the difference in the impact for the two pairs, the extent of the factor might be greater in one pair than in another. The uncertainty is even more reinforced when other theories provide other factors at an individual level which in any context may operate independently of the intermediate factors posited by the theory of socio-economic determinants of health status.

Most of the individual factors influencing the impact of HIVIAIDS have been explained by psychosocial theories or theories of individual behaviour. These theories focus on how factors such as learning factors influence risky behaviour (Bandura, 1986; Becker, 1976). According to these theories, the impact of HIVIAIDS in a group of patients will be reduced depending on their ability and willingness to learn how to avoid risky behaviour. Other theories have concentrated on beliefs, attitudes and behaviour (Rosenstock, 1974; Fishbein \& Ajzen, 1975; Ajzen, 1991). According to these theories, a person consistently behaves in relation to what they believe is right and in relation to their beliefs regarding the benefits and costs of such behaviour. The extent of the impact of HIVIAIDS in any context would depend on the extent of such beliefs, on unsafe sex for example. The model by Fisher \& Fisher $(1992,1994)$ focusing on HIVIAIDS risk behaviours and incorporating aspects of learning, attitudes and behaviour implies similar conclusions.

Some of these theories include distal factors, factors in society which influence a person's behaviour such as the person's perception of and compliance with social norms. The theories posit that individual behaviour interacts with social factors such that the impact of HIVIAIDS across socio-economic contexts depends on the extent and outcomes of such interactions. While these theories imply that the extent of the impact in a given context will depend on the preponderance of these factors and their interaction with contextual factors, their pattern and the extent of the impact of HIVIAIDS are not predictable across contexts, again implying uncertainty about the impact of contexts on HIVIAIDS outcomes

Factors used by economic theory add to the uncertainty of the impact of context on HIVIAIDS outcomes. The economic theory of risk behaviour can be traced to Becker (1976) who posited that most human behaviour can be seen as rational and utility maximizing. According to Becker (1976)'s theory, an individual balances the benefits and costs of any behaviour. Related to Becker's theory are theories by Levy (2002), Oster (2007), Bhattacharya et al. (2007), and Philipson and Posner (1993). The most commonly used of these theories in empirical work is Philipson \& Posner (1993)'s theory. This theory explains that unsafe risk behaviour takes place mainly because of mutual benefits from sexual partners who each make a rational choice, given their individual expected benefits and costs of unsafe sexual behaviour. In terms of this theory, the benefit of unsafe sexual intercourse is the avoided disutility of condoms while the costs of unsafe sex are the cost of infection ranging from pecuniary costs such as treatment costs to non-pecuniary costs such as stigma and suffering.

The way in which the economic theory explains the uncertainty of the contexts on the impact of HIVIAIDS is that the ratio of safe sex to unsafe sex is positively correlated with factors which increase the prevalence of HIVIAIDS such as location, gender, and age and not only with the individual preferences of sexual partners. In other words, the expected 
utility and costs which shape unsafe sex will depend on the characteristics of the individuals and the characteristics of the contexts in which they live with respect to the risk of infection. As these factors influence unsafe sex, so they also influence the impact of HIVIAIDS. The complexity is however, that increased awareness of the impact might or might not translate into safer sex because of other factors. Individuals consider many other aspects of life in choosing safe sex and sometimes trade off poverty against the risk of infection by accepting, for example, unsafe sex for money. The link is however, complex as some people may avoid risky behaviour because of their inability to afford the costs of treatment. These economic factors point to the fact the impact of contexts on HIVIAIDS outcomes in any context is complex and uncertain.

While economic theory focuses behavioural factors, most epidemiological theories tend to ignore the fact that increased awareness about the costs and benefits of safe behaviour cause individuals to adopt safer risk behaviour. These models state that the level of HIVIAIDS impact in context would depend mainly on the risk of infections in that context and not on individual behaviour; what the literature has termed "random sorting" (Geoffard \& Phillipson, 1996). Random sorting implies that people will choose partners irrespective of the information they have about the risk of infection. While the economic model postulates that higher prevalence and related consequences increase the costs of risk behaviour, which might discourage people demanding such behaviours, epidemiological models ignore such factors. Since the extent to which people perceive increased cost can vary from one context to another and the epidemiological model may work to varying degrees in different contexts, the impact of HIVIAIDS depending on the context is difficult to predict and therefore uncertain.

Uncertainty of the impact of contexts is compounded by the fact that different types of contexts may be interlinked to the extent that two contrasting contexts, say low prevalence area and high prevalence area may be embedded in a low socio-economic context. For instance, individual level factors in a context with low socio-economic status, which are independent of that socio-economic status might create a different prevalence context (a low prevalence context for instance) which is not in compliance with what is expected in a context with a lower socio-economic status. Figure 2, illustrates the idea.

Figure 2. Interlinking of socio-economic and epidemiological contexts

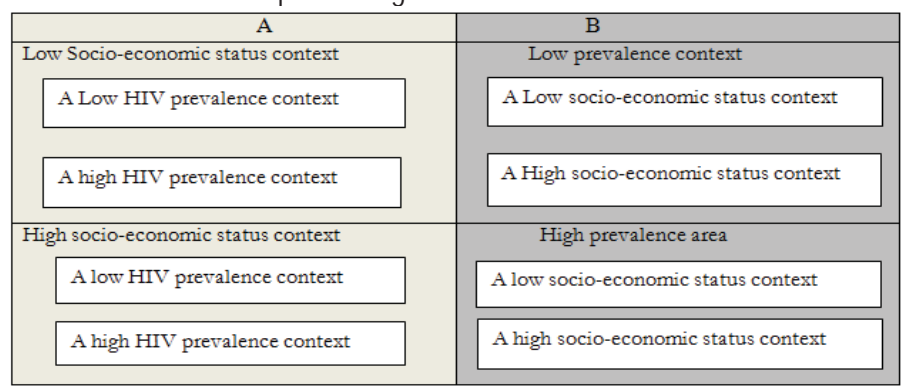

Source: Author

As Figure 2 shows, one might find two contexts with differing prevalence levels in each type of socio-economic status context (Column A of Figure 2) and a low socio-economic status and high socio-economic status context in each type of prevalence context (Column B of Figure 2) which compound the problem of uncertainty. This can be explained by other factors unaccounted for by the theory of the socio-economic determinants of health such as biological factors (see for example Habbema et al., 2005; Nicolosi et al., 1994 among many other studies). For instance, Nicolosi et al.(1994)'s evidence of greater efficiency of HIV transmission in female than in male, implies that two contexts of high prevalence and low prevalence may be sub contexts of wider context, say lower socio-economic status. The difference in prevalence in two sub-contexts may stem from the fact that one sub-context counts more female sexual partners than another subcontext.

Whether or not these factors highlighted by psychosocial, economic and epidemiological theories influence the impact of HIVIAIDS independently of the contexts in which individuals live, the patterns of these factors and the extent to which they influence the impact of HIVIAIDS across contexts cannot be easily predicted. Consequently, the effect of contexts on HIVIAIDS outcomes is uncertain and this uncertainty calls for CEA of CHIs. Another type of uncertainty that shows that the policy makers would face uncertainty without CEA of CHIs is the uncertain effectiveness of HIVIAIDS of interventions on this impact. 


\subsubsection{Uncertainty regarding effectiveness of HIV/AIDS intervention}

A context represents specific circumstances in which the impact of HIVIAIDS, in terms of new infections, AIDS cases and deaths, takes place. The effectiveness of HIVIAIDS interventions consists of the intervention's reduction of the impact and the latter reduction is done by directing specific activities to the impact. Given that many factors in a context may influence the impact of HIVIAIDS and an HIVIAIDS intervention directs activities at the impact and not necessarily at factors (at least all factors) influencing the impact, the uncertainty of the impact in the context will influence the uncertainty in the effectiveness of an HIVIAIDS intervention. Because the extent of the reduction of the impact of HIVIAIDS depends on how the intervention interacts with the circumstances influencing the impact in each context, and because the extent and patterns of these factors is uncertain, the effectiveness of HIVIAIDS interventions becomes also uncertain.

\subsubsection{Uncertainty about the costs of HIVIAIDS interventions.}

The next stage at which the uncertainty about the cost of $\mathrm{CHIs}$ materializes is through the uncertainty with which health outcomes influence the cost of HIVIAIDS interventions. The uncertainty about the cost of an HIVIAIDS intervention in a given context stems from the uncertainty of the impact of that context on health outcomes as highlighted above. The uncertain manner with which a context influences the impact of HIVIAIDS implies uncertain manner in which an HIVIAIDS intervention incur costs in a given context.

The uncertainty of costs of an HIVIAIDS intervention in a context can be explained by the link which exists between the impact of HIVIAIDS and the costs of an HIVIAIDS intervention. In fact, an HIVIAIDS interventions earmark specific activities to levels of impact of HIVIAIDS such as the level of severity (CD count below 200 for instance) and intensity (number of people infected) of HIVIAIDS. Since severity and intensity of people affected in any context is uncertain, it follows that severity-related and intensity-related activities of HIVIAIDS interventions will be uncertain. Consequently, the costs of an HIVIAIDS intervention in any context will be uncertain.

In sum, these three types of uncertainty affect the uncertainty of costs and health outcomes of $\mathrm{CHIs}$ and without CEA of CHIs, policy makers will face uncertainty with respect to how cost-effectiveness of such interventions compare. CEA of alternative HIVIAIDS interventions has been justified by the fact that policy makers could not make CE evidencebased decisions because of uncertainty about costs and health outcomes that plagued such interventions. The discussion above implies that policy makers would face uncertainty about costs and health outcomes with regard to policy making regarding $\mathrm{CHIs}$ without $\mathrm{CEA}$ of the latter $\mathrm{CHIs}$. Therefore, the case can be made for the $\mathrm{CEA}$ of $\mathrm{CHIS}$.

\subsection{The importance of the analysis argument.}

The importance of the CEA has been judged with respect to the extent to which the analysis contributed to the current evidence and then with respect to the benefit of such evidence in terms of policy implications. Generally the importance of CEA of HIVIAIDS interventions is judged on the basis of its contribution to the evidence and on the basis of the extent to which it results in policy making that resolves a problem of significant magnitude. Therefore, for the case for the CEA of $\mathrm{CHIs}$ to be made, it must be shown that without such analysis, crucial evidence would be missing and that policy makers would miss a basis from which to act in order to resolve a problem of a significant size.

\subsubsection{The contribution to the evidence}

The contribution to the CE evidence of CEA of CHIs is shown by the claim in the literature for the need of related evidence. As early as 2001, studies started putting forward claims that interventions should be designed according to the context for greater effectiveness (Grassly et al., 2001; Wegbreit et al., 2006). For instance, Grassly et al. (2001: 1121) proposed that it would be worthwhile to measure the variables describing epidemiological contexts in order to use such variables to choose interventions which best suit the context. Holtgrave \& Kelly (1996) had found that HIV prevention targeting was more cost-effective. Other literature claimed that HIVIAIDS prevention interventions are complex and made up of singular components to the extent that the most "active ingredient" needs to be identified for a particular target group in order for the intervention to be optimally effective (Bonell \& Imrie, 2001: 156). These claims insinuate that policy related to $\mathrm{CH}$ ls was needed but could not be made due to the dearth of evidence base.

CEA of CHIs derives its importance from continued shortage of the evidence base related to CEA of CHIs, implying the extent to which the latter CEA would contribute to the literature available to date. In this respect, it is worth to 
acknowledge related literature that has been conducted. In the United States, Cohen et al. (2004) conducted CEA to assess outcomes in different prevalence levels and found different estimates. Different estimates were also found by Hogan et al (2005) who compared CEA estimates of single and combined HIVIAIDS interventions in two regions of the world differing by prevalence and socio-economic levels. Kahn (1996) compared CE of HIV interventions targeting structures influencing the impact of HIVIAIDS and CE of HIV interventions targeting the impact of HIVIAIDS directly. They found that the CE of these two interventions was different. Verguet, S. \& Walsh (2010) compared the CEA of microbicides in South African, a high prevalence country, and United States (US), a low prevalence country, and found that using microbicides was more cost-effective in South Africa than it was in US.

While there is some evidence related to CEA of $\mathrm{CHIs}$, this evidence is not sufficient for policy making. For instance, country level evidence to analyse how HIVIAIDS interventions fare in different contexts of patients has not been forthcoming and there are many contexts of patients that would have been analysed for policy making such as patients with different characteristics. This deficiency of the evidence means that CEA of CHIs would contribute significantly to the CE evidence and hence the need for such an analysis.

\subsubsection{Contribution to the resolution of a major problem.}

The case for CEA of CHls can also be argued by showing that the implied efficiency would resolve a serious problem of resource shortages. The literature indicates that in 1990s and 2000s, resources committed to HIVIAIDS always fell short of the need (Alagiri et al., 2001, Kazatchkine \& Comiti, 2005). Despite efforts to close the resource gap, through pledges by United Nations Political Declarations (Joint United Nations Programme on HIVIAIDS (UNAIDS), 2012:6), resource shortages remain. Recently, the gap between resources available and needs was worsened by financial difficulties in the world which caused commitments by donor countries to multinational agencies, which are major funder of HIVIAIDS interventions; not translating into actual spending (UNAIDS 2011). The decline is likely to reverse the gains already made( Medecins sans Frontières, 2012:1). Recently UNAIDS projected that this decline could lead to a gap of $30 \%$ between resources available and resource needs by 2015 (UNAIDS, 2012:5). These shortages constitute a problem of significant magnitude. One way to deal with shortage of resources is to make sure resources that become available are used efficiently so that current health outcomes are achieved with fewer resources. In other words, ways to allocate resources in contexts and HIVIAIDS interventions according to how such interventions and contexts are efficient with the resources would need to be found and CEA of CHIs is one such ways. The importance of CEA evidence of HIVIAIDS interventions manifests in its potential to show the policy maker how best to improve efficiency, that is, to minimize costs given health outcomes to be achieved and the case for CEA of CHIs can be made on this ground.

The case for CEA of CHIs can also be made on the basis of the HIVIAIDS problem at hand. HIVIAIDS continues to be a daunting problem. Reports indicate that in 2012, about 34 million [31.4 million-35.9 million] people were living with the epidemic (UNAIDS 2012:8). In most cases, these people develop opportunistic infections resulting in a heavy burden of the epidemic. In many countries high levels of new HIV infections persist and the AIDS related death toll is still disturbing. This is the direct health burden of the epidemic without counting the indirect costs on loss of productivity for the infected, for their relatives and wider socio-economic consequences of the epidemic. As argued above, CEA of CHIs would contribute to the reduction of this problem since available resources would be allocated in contexts and in HIVIAIDS interventions to yield the highest health benefits. CEA of HIVIAIDS interventions have been conducted under the argument that the results would assist the policy makers with achieving highest health benefits given the budged. The case of CEA of CHIs is made because the results of the analysis would just assist the policy maker in a similar manner.

The case for CEA is not only supported by the magnitude of the problem to be resolved but also by the extent to which CEA of CHIs evidence-based policy would resolve this problem. In fact, evidence available makes one to believe that there would be significant differences in cost-effectiveness of contextual HIVIAIDS interventions. One study (Verguet and Walsh, 2010), comparing CE of microbicides in South Africa and the United States (US) using a hypothetical population of patients over one year, found that the estimates were quite different. Over one year, the intervention would prevent 1,908 new infections in South Africa, while it would only prevent 21 new infections in the US. In South Africa, the intervention would save US\$6,712 per infection averted, when compared with the cost of treatment (in the absence of microbicides). The study showed that the cost per infection averted by microbicides would amount to US $\$ 405,077$ in the US while the cost per infection averted was relatively less significant in South Africa.

Another study conducted comparable international contexts, South Africa and Brazil and resulted in difference in CE estimates (Dowdy et al., 2006). This study conducted CE of distributing nitrile female condom country-wide comparing 
the results of South Africa and the results of Brazil. Though these countries are comparable in in terms of socio-economic status and risk behaviours, CE results were very different. The results, over a period of three years on 1,000 hypothetical patients, were that expanding female condom use to $10 \%$ would avert 604 infections at US\$20,683 in Brazil, while in South Africa, 9,577 infections could be averted at US\$985 per infection averted.

Further evidence showing the extent to which CEA could results in huge efficiency outcomes relate to CE evidence in two regions of the world, Eastern Africa region denoted Afr-E and East Asia region denoted Sear-D (Hogan et al., 2005). The evidence as shown in Table 1, suggests huge efficiency gain that would result in CEA of CHIs. By allocating interventions on the basis of the CEA evidence, optimal use of resources would result.

Table 1. Variation in CE results across epidemiological regions

\begin{tabular}{|l|c|c|}
\hline \multicolumn{1}{|c|}{ Interventions } & \multicolumn{2}{c|}{ Average cost effectiveness ratio:\$ international per DALY averted } \\
\cline { 2 - 3 } & Afr-E & Sear D \\
\hline Individual HIV interventions & & 18 \\
Mass media & 4 & 3 \\
Peer education for sex workers & 4 & 3 \\
Peer education and treatment of STIs & $376-530$ & $432-790$ \\
School-based education & 82 & 40 \\
Voluntary counselling and testing & 34 & 310 \\
Prevention of mother- to- child transmission & $19-32$ & $20-32$ \\
Treatment of STIS & $556-2,010$ & $242-1,319$ \\
HAART & & \\
\hline
\end{tabular}

Source: Adapted from Hogan et al. (2005: 3).

These opportunities would not be revealed without CEA of CHIs. This means that without CEA of CHls, policy makers will continue to allocate resources in HIVIAIDS in contexts without due consideration of different CE of CHIs. CEA has been advanced for its potential to improve efficiency in resource allocation. Similarly, CEA of CHIs would help policy makers targeting efficiency opportunities in HIVIAIDS interventions and in contexts, making strong the case for CEA of CHIs.

Briefly, the discussion above has shown that CEA of CHIs is important in that it would contribute significantly to the CE evidence. The discussion also showed that CEA of CHIs would provide policy maker with efficiency opportunities which in turn would contribute to the solution of scarcity and other problems caused by HIVIAIDS. Without CEA of CHIs, important CE evidence related to such interventions would not be determined, strengthening the case for CEA of $\mathrm{CHIs}$. It is important however to note that the extent of the relevance of the case may vary from one country to another. The extent of the relevance of the case for South Africa is examined next.

\section{The South African Situation}

The extent to which the case for CEA of CHIs is relevant to South Africa can be shown by the degree to which South African circumstances imply uncertainty and importance of $\mathrm{CE}$ of $\mathrm{CHIs}$. The differentiated impact of the epidemic and different circumstances of patients in South Africa imply uncertainty of $\mathrm{CE}$ evidence of $\mathrm{CHIs}$ with the size of the epidemic and the shortage of resources implying that $\mathrm{CE}$ evidence of $\mathrm{CHIs}$ is important for policy making in the country.

Though South Africa is classified as generalized epidemic country, the circumstances of the epidemic are such that the impact is differentiated. The prevalence rates in South Africa vary to a great extent across provinces. Estimates in 2007 showed that prevalence among pregnant women varied considerably across provinces of the country ranging from 15.3\% in Western Cape to 38.7\% in KwaZulu Natal Province (Gouws and Qaraisha, 2010:62). Given that prevalence in women is an indicator of prevalence in the general population, the observed antenatal HIV prevalence indicate variation in prevalence across provinces. Furthermore, South Africa epidemic is also differentiated across age and gender. Projection estimates show that the age groups 15-24 years old have been the most affected than other age groups while females have suffered a great burden of the epidemic than males (Actuarial Society of South Africa, 2011). Moreover, the country has very poor rural areas and relatively very affluent urban areas. Evidence has also shown that risky behaviours are more likely to take place among the poor segment of the population although prevalence has been found to be higher in urban areas (Kalitchman and Simbayi, 2006; Kleinschmidt et al., 2007). The country counts different contexts of HIVIAIDS patients such as HIVIAIDS patients with TB co-infections as opposed to patients without co-infections, infected women who are pregnant as opposed to other non-pregnant infected women, patients with high risk of infections such as sex workers as opposed to non-high risk patients and so forth.

Bearing in mind the complexity of theoretical factors that lead to this differentiated impact, these circumstances of the epidemic imply that South Africa abounds with uncertainty on how the cost-effectiveness of HIVIAIDS interventions in these contexts of patients would compare. There is already some evidence that the impact of the epidemic in socio- 
economic contexts is uncertain. Studies found that lower socio-economic status is associated with poor HIV outcomes (Kalitchman and Simbayi, 2006; Kleinschmidt et al., 2007) while in the national survey, the urban areas were found to have more prevalence than the rural areas (Shisana and Simbayi, 2002). This uncertainty implies the need for CEA of CHIs in South Africa.

The deficiency of the evidence about how this uncertainty has been handled to assist policy making makes CEA of $\mathrm{CH}$ ls even more important. A review of the literature revealed that only a few studies related to CEA of CHIs have been conducted. Wilkinson et al. (2000) is the earlier evidence in the country which conducted CEA of PMTCT across 9 provinces of the country. The study's results showed that in fact the estimates were different across provinces. Another South African study (Vickerman et al., 2006) which conducted CEA of presumptive treatment (PPT) and usual syndromic management of STDs on contexts of patients, patients with high risk behaviours and those with low risk behaviours, found also different results across the two contexts. These CEA are not sufficient in South Africa where circumstances require more of such analyses. The deficiency of such evidence makes important the conduct of CEA of CHIs. In fact resources have already been expended in different contexts regardless of the contexts of the epidemic by policy makers because of the deficiency of this evidence. CEA of CHIs would lead to policy actions that take into account the contexts.

The importance of CE evidence from CHIs can also be argued relating the argument to the importance of the problem the analysis would help solve, the HIVIAIDS health problem. The circumstances in South Africa are such that the impact of HIVIAIDS, though declining, remains significantly high. For instance, 2013 mid-year estimates indicate that about 5.26 million people are living with HIVIAIDS and that HIVIAIDS prevalence rate in the total population is about $10 \%$ (Statistics South Africa, 2013: 4). Estimates from projection models indicate further that about 196, 000 AIDS-related deaths and 101,000 new infections are due to take place in the same year (Actuarial Society of South Africa, 2011). One of the ways to reduce further the impact with available resources is to allocate the latter in contexts and HIVIAIDS interventions such that more health benefits are achieved. But this cannot be done without CEA of CHIs which makes this analysis important in policy making in the country.

The importance of the evidence from CHIs for South Africa can further be argued by relating critical shortage of resources to the needs for such resources. it is worth to note that there has been efforts to increase resources to HIVIAIDS interventions since 2007 (Motsoaledi as cited Mbonigaba, 2013:255). However, the shortage of resources remains critical. External donors have also helped the country to some significant extent. In 2010, Global fund to fight HIVIAIDs, Tuberculosis (TB) and Malaria approved US\$302 million for a period of 5 years aimed at dealing with preventions and care activities, with specific earmarking of US\$ 196 million to AIDS medication, US\$ 33 medical male circumcision, US\$10 million to strengthen the health care system and US\$ 8 million to directly support TB programmes ( HEALTH CARE, 2010). The resources to respond to HIVIAIDs however remain very scarce despite these efforts especially that the country adopted resources intensive policies such as universal treatment of patients whose CD4 count reach a threshold CD4 count of 350. In 2012, 1.59 million people were receiving antiretroviral but this represented only about $49 \%$ of those who need it according to new policies and 2.6 million people are expected to be on highly active antiretroviral therapy by 2013-2014 (Medecins Sans Frontières, 2012:8). These resources constraints which are clearly apparent require CEA of $\mathrm{CHIs}$ implied efficiency-related policy. CEA of $\mathrm{CHIs}$ would indicate to policy makers how lower amount of resources could be used to achieve same HIV outcomes.

Not only does CEA of CHIs that inform policy decisions contribute to the solutions to the problem, but also the magnitude of such a contribution would be high as suggested by available evidence. In Table 2, variation of estimates by Wilkinson et al. (2000) of CE of PMTCT in different provinces of the country, characterized by different prevalence levels, provide an indication of efficiency opportunity implied by CEA of CHIs. The Table shows that cost per DALY averted for PMTCT varied from US\$134 to US\$ 369 making the case for CEA of CHIs worthwhile.

Table 2. Cost effectiveness results per province for the prevention of mother-to-child transmission (PMTCT) programme in South Africa

\begin{tabular}{|l|c|c|c|}
\hline Provinces & Prevented HIV infections & Cost per infection averted (R) & Cost per DALY(R) \\
\hline Gauteng & 3,809 & 6,625 & 210 \\
\hline North West & 2,357 & 6,463 & 205 \\
\hline Northern Province & 1,678 & 9,799 & 310 \\
\hline Mpumalanga & 2,507 & 5,918 & 187 \\
\hline Free State & 2,046 & 6,255 & 198 \\
\hline Northern Cape & 204 & 9,495 & 300 \\
\hline Eastern Cape & 3,171 & 7,669 & 247 \\
\hline KwaZulu-Natal & 6,769 & 4,232 & 134 \\
\hline Western Cape & 640 & 11,656 & 369 \\
\hline South Africa & 23,181 & 6,724 & 213 \\
\hline
\end{tabular}


Source: Data extracted from Table 1 of Wilkinson et al. (2000:796).

In sum, the above analysis showed that South Africa circumstances, such as the uncertainty of the impact of HIVIAIDS in contexts, the deficiency of $\mathrm{CE}$ evidence related to $\mathrm{CHIs}$, the importance of such evidence in terms the contribution to the evidence and in terms of the benefits to policy making, make every reason advanced for CEA of CHIs to be valid for South Africa. Consequently, the CEA of CHIs is relevant to South Africa to a great extent.

\section{Summary and Conclusion}

This paper argued the case for CEA of $\mathrm{CHIs}$ and the extent to which the case is relevant to South Africa. Arguing the case was motivated by the realization that the literature has mainly used uncertainty and importance argument of the CE evidence of alternative HIVIAIDS interventions to justify why CEA of such alternative was conducted.

The paper found the case to be strong after showing that uncertainty and importance argument that have underpinned the conduct of CEA of health care interventions more generally and HIVIAIDS interventions in particular could be advanced for the CEA of CHIs. Specifically, the paper found that CEA of CHIs was justified by the uncertainty underlying the costs and health outcomes of CHIs and by the importance of the evidence of CEA of CHIs for efficiencyrelated policy making. The uncertainty underlying the costs and health outcomes of $\mathrm{CHIs}$ stemmed from a multitude of contextual and individual factors likely to influence HIVIAIDS outcomes. The complexity of these factors precluded the prediction of the patterns and the extent of difference in the health outcomes of HIVIAIDS interventions in specific contexts. It was found that these health outcomes in turn had the potential to influence the costs of HIVIAIDS interventions. The argument of the importance of evidence from CEA of CHIs was founded on the fact that the evidence is deficient unearthing more evidence would contribute to the solution of the scarcity of resources and problems associated with HIVIAIDS.

With the case being strong, the implications are that CEA of CHIs should be undertaken to guide policy making. This would help the policy makers in distributing resources in HIVIAIDS interventions and contexts while taking care of efficiency and equity by making sure interventions are intensified to contexts which most benefit from them.

The case of CEA of CHIs was particularly found to be relevant to South Africa based on the country circumstances that makes a good fit for CEA of CHIs. CEA of CHIs was found to be relevant to a great extent for South Africa because its circumstances related very well to all the reasons why CEA of CHIs should be conducted. This analysis leads to the conclusion that the case for CEA of CHIs is strong one, relevant to a great extent for South Africa, and should be conducted.

\section{Acknowledgement}

The author thanks Carol Bramulge for English editing.

\section{References}

Actuarial Society of South Africa (ASSA). (2011). AIDS and demographic model of the Actuarial Society of South Africa, ASSA2008 full model.< www.actuarialsociety.org.za> (accessed November 20, 2011).

Airhihenbuwa, C. O. (2004). Culture and African contexts of HIVIAIDS prevention, care and support. Journal of Social Aspects of HIVIAIDS Research Alliance, 1(1), 4-13.

Alagiri, Collins; summers, T, Morin, S; Coates, T (2001). Global Spending on HIVIAIDS, tracking Public and Private Investments in AIDS Prevention Care and Research. Progressive Health Partners and AIDS Research Institute.

Bandura, A. (1986). Social foundations of thought and action: A Social Cognitive Theory. Englewood Cliffs, N J, Prentice Hall.

Becker, G. (1976). The economic approach to human behavior. Chicago, University of Chicago Press.

Bonell, C. \& Imrie, J. (2001). Behavioral interventions to prevent HIV infections: Rapid Evolution, Increasing Rigour, Moderate Success. British Medical Bulletin, 58, 155-170

Bowden, W.P., Rhodes, S.D. \& Jolly, C.P. (2006). Socio-cultural determinants HIVIAIDS risk and services use among immigrant Latinos in North Carolina. Hispanic Journal of Behavioral Sciences, 28 (4), 546-562

Cogneau, D \& Grimm, M. (2006). Socio-economic status, sexual behavior and differential AIDS mortality. Population Research Policy Review, 25, 393-407

Cohen, D.A., Wu, S.Y.\& Farley, T.A. (2004). Comparing the Cost-efffectiveness of HIV Prevention Interventions. Journal of Acquired Immune Deficiency Syndrome, 37,1404-1414.

Diclemente, R.J., Crosby, R.j., Kegler, M.C.(2002). Emerging Theories in Health Promotion Practice and Research Strategies for Improving Public Health. San Francisco: Jossey-Bass Publishers.

Dowdy, D.W., Sweat, M.D.\& Holtgrave, D.R. (2006). Country-wide Distribution of Nitrile Female Condom (FC2) in Brazil and South Africa: a Costeffectiveness Analysis. AIDS, 20(16), 2091-2098.

Drummond, M. F., Sculper, M. J., Torrance, G., O'Brien, B. \& Stoddant, G. L. (2005). Methods for the Economic Evaluation of Health Care Programmes. Third Edition. Oxford. Oxford University Press 
Ferry, B., Caraeil, M., Buve, A. \& Laourou, M. (2001). Comparison of key parameters of sexual Behaviours in four African urban populations with different levels of infections. AIDS, 5 (Suppl 4), S41-S60

Fisher, J.D., Fisher, W.A, Williams, S.S. \& Malloy, T.E. (1994). Empirical test of an information-motivation-behavioral skills model of AIDS preventive behaviour with gaymen and heterosexual university students. Health Psychology, 13, 238-250.

Fisher J.D. \& Fisher, W.A. (1992). Changing AIDS risk behavior. Psychological Bulletin, 111, 455-474.

Geoffard, P. Y. \& Phillipson, T. (1996). Rational epidemics and their public control. International Economic Review, 37 (3), 603-604.

Gouws, E. \& Abdool Karim, Q. A. (2010). HIV infection in South Africa: The evolving epidemic. In: HIVIAIDS in South Africa, ed. Abdool Karim, S.S \& Abdool Karim, Q.A.(eds). Second edition pp 55-73. Cambridge. Cambridge University Press.

Grassly, N. C., Garnett, G.P., Schwartlander, B., Gregson, S. \& Anderson, R. M. (2001). The effectiveness of HIV prevention and the epidemic context. Bulletin of the World Health Organisation, 79(12), 1121-1132.

Habbema, J.D \& Hayes, R. J. (2005). Determinants of the impact of sexually transmitted infections on prevention of HIV infections: A Synthesis of evidence from Mwanza, Rakai, and Masaka Intervention. The Journal of Infectious Diseases, 191 (Suppl 1), S168-S175.

HEALTH CARE (2010). Global Fund helps SA fight HIVIAIDS<http://www.south africa.info/about/health/globalfundgrant_201210.htm

Hogan, D. R., Balthussen, R., Hayashi, C., Laurer, J. A. \& Salomon, J. A. (2005). Cost-effectiveness analysis of strategies to combat HIVIAIDS in developing countries. Biomedical Journal, 2, 123-135.

Holtgrave, D. R. \& Kelly, J. A. (1996). Preventing HIVIAIDS among high-risk urban women: The cost-effectiveness of behavioural group interventions. American Journal of Public Health, 86, 1442-1445.

Kalitchman, S.C \& Simbayi, L.C. (2006). Association of Poverty, Substance Use, and HIV Transmission Risk Behaviors in Three South African Communities. Social Science and Medicine, 62(7), 1641- 1649.

Kazatchkine, M.D and Comiti, C.(2005). Global Resources for HIVIAIDS. http://www.globalresourcesforhiv .pdf (accessed 12/05/2013)

Kahn, J.G. (1996). The cost-effectiveness of HIV Prevention Targeting: How much More Bangs for the Buck? American Journal of Public Health, 86 (12), 1709-1711.

Kleinschmidt, I., Pettifor, A., Morris, N., MacPhail, C. \& Rees, H. (2007): Geographic distribution of the Human Immunodeficiency Syndrome in South Africa. American Journal of Tropical Medicine, 77 (6), 1163-1169

Levy, A (2002), A Lifetime Portfolio of Risky and Risk-free Sexual Behaviour and the Prevalence of AIDS. Journal of Health Economics, 21(6), 993-1007.

Marmot, M. \& Wilkinson, R. (2005). Social determinants of health. Oxford. Oxford University Press

Mbonigaba. J (2013). Constraints on the potential effectiveness of HIVIAIDS early treatment policy in South Africa. In: Global HIVIAIDS politics, policy, and activism: persistent challenges and emerging issues: vol.2. policy and policymaking.Chapter12.smith, r.a. (ed.).(2013), Santa Barbara, Ca: Praeger.

Medecins sans Frontieres ( 2012). Reversing HIVIAIDS? How advances are being held back by funding shortages. AIDS Briefing. < http://www.aidbriefing_ reversing AIDS ENGFeb2012update. pdf>

Muennig, P. (2008). Cost-effectivenes Analysis in Health, A Practical Approach. Second edition. San Francisco. Jossey-Bass.

Nicolosi, A.,Correa leit, M.L., Musiccom, M., Aric, C., Gavazzeni, G.\& Lazzarin, A. (1994). The efficiency of male-to-female and female-to-male sexual transmission of the human immunodeficiency virus: A Study of 730 stable couples. Epidemiology, 5(6), 570-575.

Oster, E. (2007). HIV and sexual behaviour change: Why Not Africa?, NBER Working Paper, No. 13049

Bhattacharya, C., Bunzel, H., \& Qiao, X. (2007). Unsafe sex, AIDS and development, Staff General Research Papers, No. 12832, Department of Economics, lowa State University

Ojteg, K. (2008). Socio-economic determinants of HIV in Zambia: A district level analysis. School of Economics and Management. Lund University. Master's Thesis.

Philipson, T.\&Posner, R.(1993). Privates Choices and Public Health: The AIDS Epidemic in an Economic Perspective. Cambridge. Havard University Press.

Shisana, O. \& Simbayi, L.C. (2002). Nelson Mandela/HSRC Study of HIVIAIDS: South African national HIV prevalence, behavioral risks and mass media: Household survey 2002: Cape Town. HSRC.Trials.Snelling, D., Rasugu,

Snelling, D., Omariba, R., Hong, R.D.W., Georgiades, K., Racine, Y \& Boyle, M.H. (2007). HIVIAIDS knowledge, women's education, epidemic severity and protective sexual behaviour in low- and middle-income countries. Journal of Biosocial Science, 39 (3), p $421-442$

Spanish Commission for Reducing Health Inequalities. (2007). Socio-economic determinants of Health. <http://www.instituteofhealthequity.org /projects/spanish-commission-to-reduce-inequalities-in-health> (accessed March 10, 2012)

Statistics South Africa (2013). Mid-year Population estimates, Statistical Release. Pretoria. <http://www.statssa.gov.za/publications /P0302 /P03022013.pdf> (accessed May25, 2013

Tiruneh, G (2009). Determinants of HIVIAIDS prevalence in Africa: Do cultural variations matter? Midsouth Political Science Review, 10, 103-123

UNAIDS (2011). Global Report:UNAIDS Report on the Global AIDS Epidemic 2011. Geneva, UNAIDS.

UNAIDS (2012). UNAIDS Report on the Global AIDS Epidemic. Geneva.

Valentine, N. \& Solar, O. (2011).The conceptual framework for social determinants of health. Which theory is the basis for tool for health impact assessment? PP Presentation. Department of Ethics, Equity, Trade and Human Rights / WHO. Accessed at si.easp.es/../.VandePierre

Verguet, S. \& Walsh, J.A. (2010). Vaginal Microbicides Save Money: A Model of Cost-effectiveness in South Africa and the USA. Sexually Transmitted Infections, 86 (3), 212-216

.Vickerman, P., Terris-Prestholt, F., Delany, S., Kumaranayake, L., Rees, H. \& Watts, C. (2006). Are Targeted HIV Prevention Activities Still Cost-Effective in High Prevalence Settings? Results From an STI Treatment Interventions For Sex Workers in Hillbrow, South Africa. Sexually Transmittable Diseases, 33 (10), S122-S132

Vickerman, P; Watts, C; Delany, Alany, M., Rees, H., Heise, L. (2006). The importance of context: Model projections on how microbicide impact could be affected by the underlying epidemiological and behavioural situation in two African settings. Sexually Transmitted Diseases, 33, 397-495

Wegbreit, J., Bertozzi, S., DeMaria, L.M., \& Padian, N.S. (2006). Effectiveness of HIV prevention strategies in resource-poor countries: Tailoring the Intervention to the Context. AIDS 20, 1217-1235

Wilkinson, D., Floyd, K. \& Gilks, F. C. (2000). National and provincial estimated costs and cost-effectiveness of a programme to reduce mother-to-child HIV transmission in South Africa. South African Medical Journal, 90 (8), , 794-798.

Zanakis, S.H., Alvaez, C., \& Li, V. (2006). Socio-economic Determinants of HIV Pandemic and nations Efficiencies. European Journal of Operational Research, 176:1811-1838 